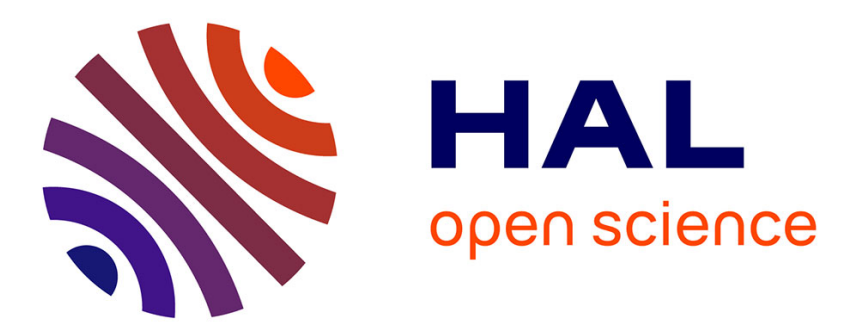

\title{
Evaluation of Medical Gestures Based on a Global Performance Index
}

\author{
Richard Moreau, Victor M. Ochoa Mayorga, Minh Tu Pham, Pierre
}

Boulanger, Olivier Dupuis

\section{- To cite this version:}

Richard Moreau, Victor M. Ochoa Mayorga, Minh Tu Pham, Pierre Boulanger, Olivier Dupuis. Evaluation of Medical Gestures Based on a Global Performance Index. 31st IEEE EMBC, Sep 2009, Minneapolis, MN, United States. pp.5854 - 5857, 10.1109/IEMBS.2009.5334410 . hal-00416218

\section{HAL Id: hal-00416218 \\ https://hal.science/hal-00416218}

Submitted on 23 Apr 2010

HAL is a multi-disciplinary open access archive for the deposit and dissemination of scientific research documents, whether they are published or not. The documents may come from teaching and research institutions in France or abroad, or from public or private research centers.
L'archive ouverte pluridisciplinaire HAL, est destinée au dépôt et à la diffusion de documents scientifiques de niveau recherche, publiés ou non, émanant des établissements d'enseignement et de recherche français ou étrangers, des laboratoires publics ou privés. 


\title{
Evaluation of Medical Gestures Based on a Global Performance Index
}

\author{
R. Moreau, V. Ochoa, M.T. Pham, P. Boulanger and O. Dupuis
}

\begin{abstract}
This paper presents a method to evaluate medical gestures. The objective is to objectively assess a gesture carried out by novice doctors. The proposed method is based on the study of the curvature of the 3D gesture and provide a global performance index for one manipulation. The study of the number of peaks on the curvature indicates if the gesture is smooth or not. The application is the obstetric gestures linked to the forceps use but the method can be applied to different gestures without loss of generality. Seven residents carried out 30 forceps blade placements. The results clearly show a difference between the gestures carried out. This highlights the difficulty of the gesture according to the fetal head presentation.
\end{abstract}

\section{INTRODUCTION}

Nowadays, medicine residents mainly train in the operative room. They are evaluated by experienced doctors who determine if a resident has acquired sufficient experience to be autonomous. It is always awkward to evaluate a resident just by watching the gestures he carried out in the operative room. Moreover this problem becomes more complex if the gestures is carried out inside the patient body which is often the case in medical procedure. Another issue also occurs as the training is carried out on real patients. To avoid situations, which could be dramatic to the patients, several medical simulators have been developed over the last decades to offer an alternate training: simulator training. Some companies offer medical simulators to hospitals (Simulaids ${ }^{1}$, Laerdal $^{2}$, Simulution ${ }^{3}$, Limbandthings ${ }^{4}$, Gaumard ${ }^{5}$, etc.). All these simulators provide an anthropomorphic interface, which allows doctors to find the same landmarks than in normal operative conditions. Their main drawback lies on their lack of haptic and visual feedback to allow the simulators to be sufficiently realistic to provide useful training, and above all, they do not provide an objective assessment to quantify the gestures.

To complete these simulators, several researchers in collaboration with medical doctors developed simulators for different fields. For instance, simulators are now available for obstetricians [1]-[4], hysteroscopic procedures [5], otologic surgery [6], endoscopic surgery [7], anesthesiologists [8],

R. Moreau are M.T. Pham are with the Ampere lab., INSA-Lyon, Université de Lyon, F-69621, France richard.moreaulinsa-lyon. fr

V. Ochoa, and P. Boulanger are with the AMMI lab., Dpt of Computing Science, University of Alberta, T6G2E8, Canada pierreb@cs.ualberta.ca

O. Dupuis is with the CHU Lyon Sud, 69495 Pierre-Bénite, France olivier. dupuisechu-lyon.fr

${ }^{1}$ http://www.simulaids.com/

${ }^{2}$ http://www.laerdal.com/

${ }^{3}$ http://www.simulution.com/

${ }^{4}$ http://www.limbandthings.com/

${ }^{5}$ http://www.gaumard.com/ palpatory diagnostics [9], suture/ligature training [10], physiotherapists [11] (this list is not exhaustive).

The objective of all these simulators is to offer residents new training methods to acquire a first experience without any risks for the patients. Their aim is not to be a substitute to classical training in the operative room but to complement it. In order to be efficient, these simulators need to provide an assessment of the trained gestures. Thus, residents can determine their weakness and eradicate their main defaults.

The obstetrics gesture we want to analyze consists of forceps placement. Forceps are the obstetrics instruments which ensure the most physiological childbirth i.e. their particular design respect the cephalic curvature and the pelvic curvature which allow to exert an effort in the same direction than the natural expulsive forces of the mother. Forceps consists of two parts, called blade, which are assembled once they are correctly place. Fig. 1 represents the forceps mainly use in the "Hospices Civils de Lyon (HCL)" and their final position around the fetal head inside the maternal pelvis.

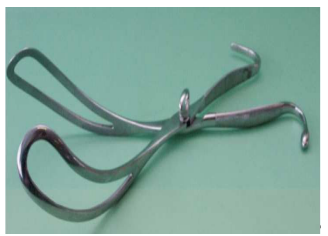

(a) Levret Forceps

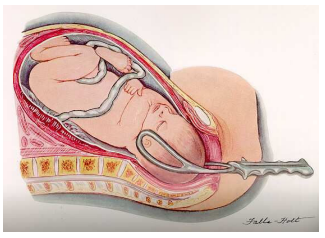

(b) Forceps around the fetal head
Fig. 1. Picture of the Levret forceps and scheme of their final position.

In this paper, we proposed thus a method to ensure an objective assessment of gestures using forceps. It could be applied to analyze different gestures. However, we especially focus on the obstetric gestures linked to the forceps use. The BirthSIM simulator has been used to record residents in obstetrics. The paper is organized in three parts. The first part is dedicated to the description of the material used: the BirthSIM simulator and the method we propose. The second part presents the results we obtained and finally the last part consists of a discussion followed by a conclusion.

\section{Materials AND Methods}

\section{A. The BirthSIM Simulator and Its Instrumented Forceps}

The BirthSIM simulator [12] has been used to allow residents to train to place the forceps blades. The simulator consists of a realistic manikin to reproduce accurately the maternal pelvis and the fetal head. This manikin is instrumented with an electro-pneumatic component to simulate the dynamic process of a childbirth. A forceps has been 
instrumented with two (one in each blade) electromagnetic sensors with the ability to measure six degree of freedom [4]. With such instrumentation, it is possible to record forceps blade paths in order to analyze and compare them. For this study only the forceps displacement is recorded, the extraction manipulation will be a part of a new experimental protocol.

\section{B. Analysis of 3D Trajectories}

In collaboration with obstetricians, we define these criteria to study the gestures:

- The analysis has to be time independent because the the time needed to place the forceps is not crucial;

- The whole trajectory has to be taken into account, an uncorrect movement can lead to dramatic consequences for the fetus and/or the patient;

- The position and the orientation of the blades are crucial and thus have to be analyzed.

To analyze human gestures and in particular medical gestures, we proposed to compare the curvature of the gestures. Position data are first expressed according to their chord length to guarantee time independence. The curvature which corresponding to the second derivative norm is then computed [13]. Concerning orientation data, they are expressed in the unit quaternion space and the curvature of the trajectory on the unit sphere is then computed [14]. Quaternion convention have been preferred to Euler convention due to their advantages [15]:

- Geometrical interpretation is easier using quaternions than Euler angles. Every rotation can be expressed by a quaternion which represent the rotation angle about the rotation axis.

- Contrary to Euler angles, no order of rotation has to be respected.

- Such quaternions represent the rotation angle about the rotation axis, interpolation is easier.

- The representation of rotation is more compact using quaternions than Euler angles.

- No gimbal lock phenomenon.

Studying the curvature allows to know how smooth the gesture is. In fact, the brusque variations of the curvature mean that the operator suddenly changed the direction of its instruments which can damage the parturient or the fetus. The residents therefore have to learn to reduce them. These brusque variations can be detected by studying the high values on the curvature. To detect them, we choose a threshold and points beyond this threshold are considered as potentially dangerous. We can thus detect the number of times the operator has brusquely changed its instrument direction. To determine the threshold, an arbitrary value is chosen. This value corresponds to a significant change in the direction.

As position data and orientation data have to be both analyzed, we decide to gather their curvatures which will be studied to assess the gestures carried out. The assessment consists of evaluating the number of peaks on the curvature and comparing the curvature of novices and experienced doctors by correlation. The correlation is calculated using the Pearson correlation coefficient which is computed as follows.

For two curvature vectors $\vec{A}$ and $\vec{B}$ this coefficient $r_{p r}$ is given by:

$$
r_{p r}=\frac{\sum_{i=1}^{i=n}\left(A_{i}-\overline{A_{m}}\right)\left(B_{i}-\overline{B_{m}}\right)}{\sqrt{\sum_{i=1}^{i=n}\left(A_{i}-\overline{A_{m}}\right)^{2} \sum_{i=1}^{i=n}\left(B_{i}-\overline{B_{m}}\right)^{2}}}
$$

with :

$A_{i}$ is the $i^{t h}$ component of the first curvature vector; $\overline{A_{m}}$ is the average of the components of $\vec{A}$;

$B_{i}$ is the $i^{t h}$ component of the second curvature vector; $\overline{B_{m}}$ is the average of the components of $\vec{B}$.

\section{Experimental Protocol}

In collaboration with the Hospices Civils de Lyon (HCL) seven residents were trained on the BirthSIM simulator. The simulator training is supervised under the authority of an expert obstetrician who is the instructor. An expert obstetrician is defined as an obstetrician with at least ten years of experience, and who have used forceps in more than $80 \%$ of his instrumental interventions. The fetal head is positioned according to the ACOG (American College of Obstetrics and Gynecology) classification [16]. The presentation is cephalic, that is to say the head comes in first. Two presentations are studied: $\mathrm{OA}+4$ (Occiput Anterior location and station $+4 \mathrm{~cm}$ from the ischial spines plan) and LOA +5 (Left Occiput Anterior location and station $+5 \mathrm{~cm}$ from the ischial spines plan). Fig. 2 represents the different cephalic presentations.

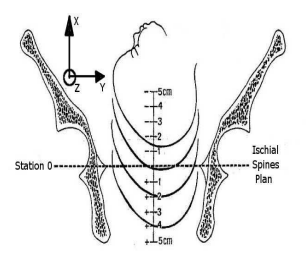

(a) Station

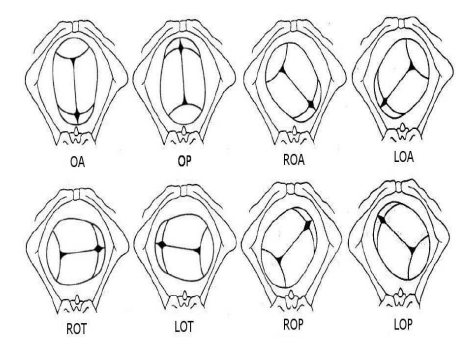

(b) Location
Fig. 2. The different presentations of the fetal head according to the ACOG.

Each resident carried out 30 forceps blade placements for both presentations. It approximately represents the number of forceps blade placement that they may assist during their three years of residency.

\section{RESULTS}

Concerning $\mathrm{OA}+4$ presentation, forceps blades have symmetric trajectories around the fetal head as shown on Fig. 3. On this figure, only the position are represented and the $\mathrm{X}$-axis correspond to the cranio-caudal axis, the $\mathrm{Y}$-axis is transversal and the Z-axis is vertical as shown on Fig. 2. On Fig. 3 and 4, the pelvis and the fetal are represented to better 
understand the trajectories of the blades, however it is only a representation, it does not correspond to their real position.

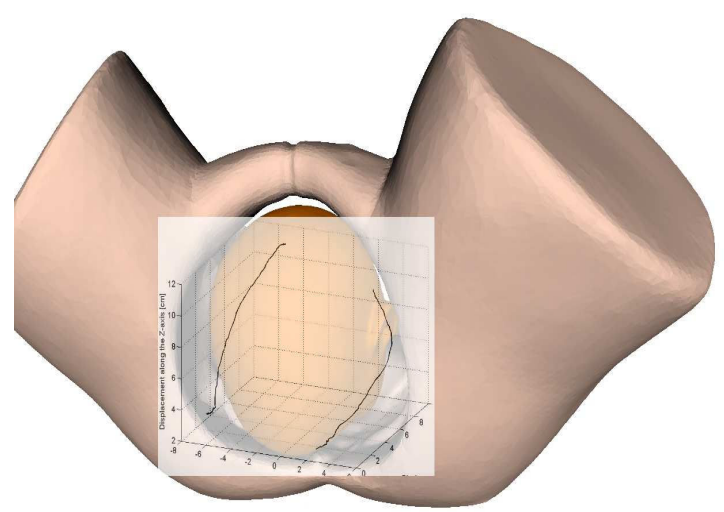

Fig. 3. Forceps blades trajectories of an expert for $\mathrm{OA}+4$ presentation

Concerning LOA+5 presentation, forceps blades have asymmetric trajectories around the fetal head as shown on Fig. 4.

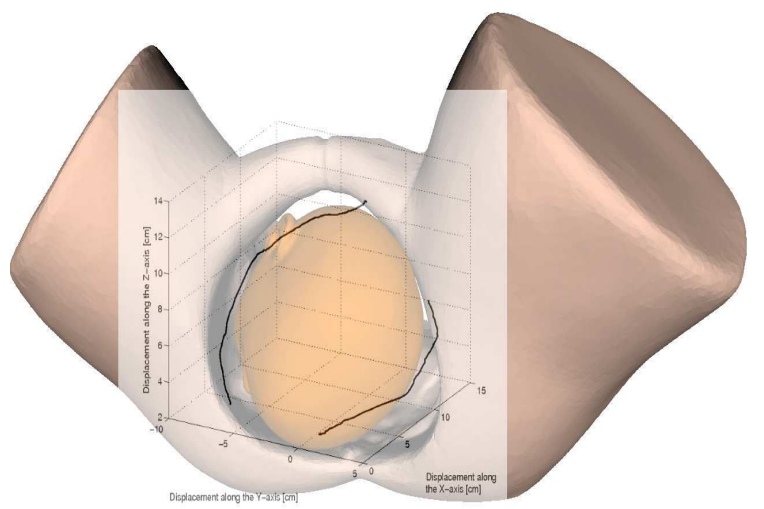

Fig. 4. Forceps blades trajectories of an expert for LOA +5 presentation

On this figure, only the position are represented and the right forceps have to respect an important rotation in order to circumvent the fetal head and to reach its final position (behind the fetal ear).

To visualize the orientation data of the forceps during their placement, they are expressed on an unit sphere which represents the quaternion space. Fig. 5 represents the orientation of both forceps blades. On this figure, we can have the same conclusion than the position study: both blades have symmetrical trajectories. Indeed for $\mathrm{OA}+4$ presentation, the forceps are initially vertical: the forceps tips inside the vulva and against the fetal head, then the obstetrician displaces the blade along the fetal head to reach its final position: along the fetus jaw and with a horizontal position.

Concerning the $\mathrm{LOA}+5$ presentation, orientation data are represented on Fig. 6. We can observe that the trajectories are different. This is due to the orientation of the fetal head, the left blade has a small angular amplitude contrary to the right blade which is submitted to a high variation concerning its orientation.

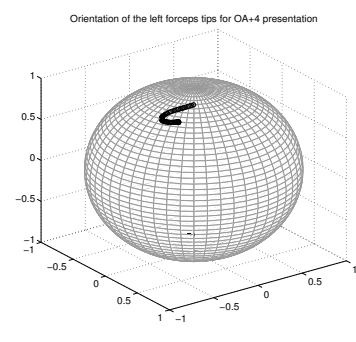

(a) Left Forceps

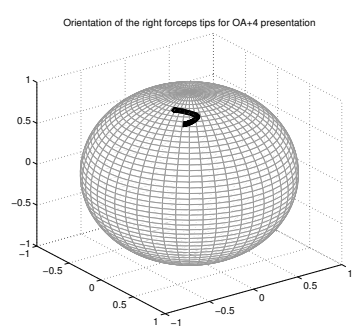

(b) Right Forceps
Fig. 5. Orientation of the forceps for $\mathrm{OA}+4$ presentation

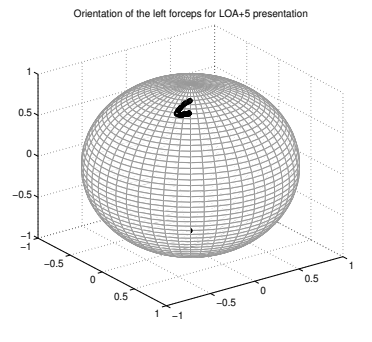

(a) Left Forceps

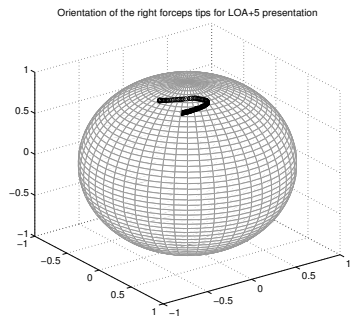

(b) Right Forceps
Fig. 6. Orientation of the forceps for $\mathrm{LOA}+5$ presentation

The next step is the study of the curvature for the position and the orientation of both blades. As indicated in II-B, the studied curvature consists of both curvatures: the position and the orientation as they are both crucial during the forceps blade placement. Table I gathers the results concerning the number of peaks on this curvature. The results presented in this table correspond to the average over all the attempts of all residents. Concerning the expert, he carried out 5 forceps blade placements.

TABLE I

NUMBER OF PEAKS ON THE GLOBAL CURVATURE

\begin{tabular}{|c|c||c|c||c|c||}
\hline \multicolumn{2}{|c||}{} & \multicolumn{2}{c||}{$\begin{array}{c}\text { Presentation } \\
\text { OA+4 }\end{array}$} & \multicolumn{2}{c||}{$\begin{array}{c}\text { Presentation } \\
\text { LOA+5 }\end{array}$} \\
\cline { 3 - 6 } \multicolumn{2}{|c||}{} & $\begin{array}{c}\text { Left } \\
\text { Forceps }\end{array}$ & $\begin{array}{c}\text { Right } \\
\text { Forceps }\end{array}$ & $\begin{array}{c}\text { Left } \\
\text { Forceps }\end{array}$ & $\begin{array}{c}\text { Right } \\
\text { Forceps }\end{array}$ \\
\hline \multirow{2}{*}{$\begin{array}{c}\text { Number } \\
\text { of peaks }\end{array}$} & Residents & 36 & 46 & 45 & 61 \\
\cline { 2 - 6 } & Experts & 17 & 18 & 22 & 23 \\
\hline
\end{tabular}

The expert does not have any difficulty to smoothly place the forceps whatever the fetal head presentation is. Concerning the residents they clearly have difficulty to ensure a smooth gestures and in particular for the right blade when the fetal head is in LOA +5 presentation. This is due to the fact that the blade has to respect an important rotation to reach its final position which requires a certain experience.

To evaluate residents, the curvature is compared to the expert one by correlation. Table II gathers the correlation results. The correlation coefficient is given in $\%$ and it corresponds to the average of the 30 forceps blade placements carried out by the seven residents.

This table highlights the difficulty that residents have to carry out a correct gestures. The correlation coefficient is 
TABLE II

Correlation CoEFFICIENT OF THE CuRvature In \% FOR THE RESIDENTS.

\begin{tabular}{|c||c|c||c|c||}
\hline \multicolumn{1}{|c||}{} & \multicolumn{2}{c||}{$\begin{array}{c}\text { Presentation } \\
\text { OA+4 }\end{array}$} & \multicolumn{2}{c|}{$\begin{array}{c}\text { Presentation } \\
\text { LOA+5 }\end{array}$} \\
\cline { 2 - 5 } & $\begin{array}{c}\text { Left } \\
\text { Forceps }\end{array}$ & $\begin{array}{c}\text { Right } \\
\text { Forceps }\end{array}$ & $\begin{array}{c}\text { Left } \\
\text { Forceps }\end{array}$ & $\begin{array}{c}\text { Right } \\
\text { Forceps }\end{array}$ \\
\hline Correlation coefficient & 15 & 16 & 30 & 10 \\
\hline
\end{tabular}

globally low, and it is even lower for the right forceps for LOA+5 presentation whereas the left forceps for this presentation has a higher value.

\section{DISCUSSION AND CONCLUSION}

This paper presents our work on the analysis of medical gesture based on the study of the curvature. The curvatures of the position and the orientation data are computed. This allows to obtain a global performance index thanks to the computation of a correlation coefficient with respect to the results obtained with an expert. The peaks on the curvature reflect how smooth the trajectories are. To avoid consequences on the mother and the fetus, which could be dramatic, smoother the trajectory is and safer the gesture is.

Both tables indicate different levels of difficulty on the forceps blade placement. It above all highlights the fact that the current training is not sufficient for obstetricians to gain experience in handling the forceps. A simulator training may improve their skills and allow them to riskfree train. A longer study may be carried out comparing two groups of residents: one will be trained on the BirthSIM simulator while the other one will not. Then to compare both groups only a subjective evaluation may be carried on about the self confidence residents have with forceps because it is impossible to measure the gestures in the childbirth room.

Concerning the method presented in this paper, it should be applied to another gesture to estimate its efficiency. Anyway the study of the curvature informs us how smooth is the trajectory. The study of the peaks on the curvature indicates when the obstetrician carried out a brusque change in its gesture which may cause damages during a real delivery.

This preliminary study shows the main differences between forceps blade placements according to the presentation of the fetal head. It should be improved by studying more precisely the right blade movement while the fetal head is in LOA location. This description may help the residents to understand the good gesture they have to acquire in order to ensure a safe forceps extraction.

To complete this study it will be interested to use the visualization interface available on the BithSIM simulator. The next experimental protocol should include the method proposed in [17]. The progression of residents trained on the simulator may then be analyzed using the method presented in this paper. Finally, another alternative is to extend the proposed method to a study on different gestures including different kinds of data, which could be not only positions and orientations data but also forces and torques data.

\section{REFERENCES}

[1] R. Lapeer, M. S. Chen, and J. Villagrana. Simulating obstetric forceps delivery in an augmented environment. In Augmented environments for Medical Imaging including Augmented Reality in Computer-aided Surgery (AMI ARCS'04), Rennes, France, 2004.

[2] T. Sielhorst, T. Obst, R. Burgkart, R. Riener, and N. Navab. An augmented reality delivery simulator for medical training. In Augmented environments for Medical Imaging including Augmented Reality in Computer-aided Surgery (AMI ARCS'04), pages 11-20, Rennes, France, 2004.

[3] E. J. Kim, P. Theprungsirikul, M. K. McDonald, E. D. Gurewithsch, and R. H. Allen. A biofidelic birthing simulator. IEEE Engineering in Medicine and Biology Magazine, 24(6):34-39, Nov.-Dec. 2005.

[4] R. Moreau, M.T. Pham, R. Silveira, T. Redarce, X. Brun, and O. Dupuis. Design of a new instrumented forceps: Application to safe obstetrical forceps blade placement. IEEE Transactions on Biomedical Engineering, 54(7):1280-1290, July 2007.

[5] F. Lim, I. Brown, R. Mccoll, C. Seligman, and A. Alsaraira. Hysteroscopic simulator for training and educational purposes. In $28^{\text {th }}$ Annual International Conference of the IEEE Engineering in Medicine and Biology Society, (EMBC '06), pages 1513-1516, Aug. 2006.

[6] R. Grunert, G. Strauss, H. Moeckel, M. Hofer, A. Poessneck, U. Fickweiler, M. Thalheim, R. Schmiedel, P. Jannin, T. Schulz, J. Oeken, A. Dietz, and W. Korb. Elephant - an anatomical electronic phantom as simulation-system for otologic surgery. In 28th Annual International Conference of the IEEE Engineering in Medicine and Biology Society, (EMBC '06), pages 4408-4411, Aug. 2006.

[7] M. Karouia, P. Arhets, and Y. Aigrain. A novel design of endoscopic surgery training simulator. In $35^{\text {th }}$ International Symposium on Robotics (ISR'04), page 66, Paris, France, 23-26 March 2004.

[8] L. Hiemenz, J. McDonald, D. Stredney, and D. Sessanna. A physiologically valid simulator for training residents to performan epidural block. In Proceedings of the 1996 Fifteenth Southern Biomedical Engineering Conference, pages 170-173, March 1996.

[9] M. Y. Chen, R. L. Williams II, R. R. Conatser JR., and J. N. Howell. The virtual movable human upper body for papatory diagnostic training. In Digital Human Modeling for Design and Engineering, DHM'06, Lyon, France, 2006.

[10] N. Oshima, M. Aizudding, R. Midorikawa, J. Solis, Y. Ogura, and A. Takanishi. Developement of a suture/ligature training system designed to provide quantitative information of the learning progress of trainees. In IEEE International Conference on Robotics and Automation (ICRA'07), pages 2285-2291, Roma, Italy, 10-14 April 2007.

[11] L. Marechal, C. Barthod, J. Lottin, G. Gautier, and J.-C. Jeulin. Measurement system for gesture characterization during chest physiotherapy act on newborn babies suffering from bronchiolitis. In $29^{\text {th }}$ Annual International Conference of the IEEE Engineering in Medicine and Biology Society, (EMBC '07), pages 5770-5773, Lyon, France, August 2007.

[12] R. Silveira, M. T. Pham, T. Redarce, M. Betemps, and O. Dupuis. A new mechanical birth simulator: BirthSIM. In IEEE/RSJ International Conference on Intelligent Robots and Systems, (IROS'04), volume 4, pages 3948-3953, 28 Sept.-2 Oct. 2004.

[13] R. Moreau, V. Ochoa, M.T. Pham, P. Boulanger, T. Redarce, and O. Dupuis. Evaluation of obstetric gestures: An approach based on the curvature of 3-D positions. In 29th Annual International Conference of the IEEE Engineering in Medicine and Biology Society (EMBC'07), pages 3634-3637, Lyon, France, Aug. 2007.

[14] R. Moreau, V. Ochoa, M.T. Pham, P. Boulanger, T. Redarce, and O. Dupuis. Evaluation of obstetric gestures: An approach based on the curvature of quaternions. In 30th Annual International Conference of the IEEE Engineering in Medicine and Biology Society (EMBC'08), pages 3430-3433, Vancouver, Canada, August 2008.

[15] A. J. Hanson. Visualizing Quaternions. The Morgan Kaufmann Series in Interactive 3D Technology. Morgan Kaufmann Publishers, 2006. ISBN 0-12-088400-3.

[16] G. Cunningham, L. Gilstrap, K. Leveno, S. Bloom, J. Hauth, and K. Wenstrom. Williams Obstetrics. the McGraw-Hill Companies, $22^{\text {nd }}$ edition, 2005. ISBN 0071413154.

[17] R. Moreau, M.T. Pham, T. Redarce, and O. Dupuis. A new learning method for obstetric gestures using the BirthSIM simulator. In IEEE International Conference on Robotics and Automation (ICRA'07), pages 2279-2284, Roma, Italy, 10-14 April 2007. 